\title{
Characterization of NADP-dependent L-arginine dehydrogenase as a novel amino acid dehydrogenase and its application to an L-arginine assay
}

\section{Toshihisa Ohshima ( $\nabla$ toshihisa.oshima@oit.ac.jp)}

Osaka Institute of Technology: Osaka Kogyo Daigaku https://orcid.org/0000-0003-3268-725X

\section{Taketo Ohmori}

Osaka Institute of Technology: Osaka Kogyo Daigaku

Masaki Tanaka

Osaka Institute of Technology: Osaka Kogyo Daigaku

\section{Research Article}

Keywords: L-arginine dehydrogenase, Pseudomonas veronii, molecular cloning, NADP-dependent amino acid dehydrogenase, L-arginine determination

Posted Date: January 13th, 2022

DOl: https://doi.org/10.21203/rs.3.rs-860736/v2

License: (c) (1) This work is licensed under a Creative Commons Attribution 4.0 International License.

Read Full License 
1 Journal: Appl. Biochem. Biotechnol. Original Investigation

2

3 Title: Characterization of NADP-dependent L-arginine dehydrogenase as a novel amino acid

4 dehydrogenase and its application to an L-arginine assay

5

6 Authors: Toshihisa Ohshima ID $\cdot$ Masaki Tanaka $\cdot$ Taketo Ohmori

7 Department of Biomedical Engineering, Faculty of Engineering, Osaka Institute of Technology,

8 5-16-1 Omiya, Asahi-ku, Osaka 535-8585, Japan.

9

10

11

${ }^{*}$ Corresponding author: Toshihisa Ohshima

12

Tel: +81-6-6954-4608, fax: +81-6-6954-4649, e-mail: tohishisa.oshima@oit.ac.jp

13 ORCID of Toshihisa Ohshima: https://orcid.org/0000-0002-7751-7695

14

15

16

17 


\section{$1 \quad$ Abstract}

2 L-Arginine dehydrogenase (L-ArgDH, EC 1.4.1.25) is an amino acid dehydrogenase which catalyzes

3 the reversible oxidative deamination of L-arginine to the oxo analog in the presence of NADP.

4 Although the enzyme activity is detected in the cell extract of Pseudomonas aruginosa, the purification

5 and characterization of the enzyme have not been achieved to date. We here found the gene

6 homolog of L-ArgDH in genome data of Pseudomonas veronii and succeeded in expression of $P$.

7 veronii JCM11942 gene in E. coli. The gene product exhibited strong NADP-dependent L-ArgDH

8 activity. The crude enzyme was unstable under neutral $\mathrm{pH}$ conditions, but was markedly stabilized by

9 the addition of $10 \%$ glycerol. The enzyme was purified to homogeneity through a single Ni-chelate

10 affinity chromatography step and consisted of a homodimeric protein with a molecular mass of about

$1165 \mathrm{kDa}$. The enzyme selectively catalyzed L-arginine oxidation in the presence of NADP with maximal

12 activity at $\mathrm{pH}$ 9.5. The apparent $K_{\mathrm{m}}$ values for L-arginine and NADP were 2.5 and $0.21 \mathrm{mM}$,

13 respectively. The nucleotide sequence coding the enzyme gene (was determined and the amino

14 acid sequence was deduced from the nucleotide sequence. As an application of the enzyme, simple

15 colorimetric microassay for L-arginine using the enzyme was achieved.

17 Key words L-arginine dehydrogenase, Pseudomonas veronii, molecular cloning, NADP-dependent amino acid dehydrogenase, L-arginine determination 
1

2

4

5

6

7

8

9

deamination of amino acids to their corresponding oxo analogs in the presence of $\mathrm{NAD}(\mathrm{P})$, have

18 been found so far. Among them, glutamate dehydrogenase, leucine dehydrogenase, alanine

19

dehydrogenase and phenylalanine dehydrogenase have all been well characterized and are widely
Masaki Tanaka

masaki01142580@i.softbank.jp

\section{Taketo Ohmori}

Taketo.omori@oit.ac.jp

Department of Biomedical Engineering, Faculty of Engineering, Osaka Institute of Technology,

5-16-1 Omiya, Asahi-ku, Osaka 535-8585, Japan.

\section{Introduction}

More than twenty different amino acid dehydrogenases (EC 1.4.1.-), catalyzing the reversible

used for industrial asymmetric production of L-amino acids and their analogs [1-4], which then serve 
1 as important nutrients and pharmaceutical compounds. In addition, amino acid dehydrogenases have

2 been used for sensing amino acids in foods and blood and for enzyme assays [1, 5]. For those

3 reasons, novel amino acid dehydrogenases have the potential to be highly useful [2]. Among these

4 enzymes, $\mathrm{NAD}(\mathrm{P})$-dependent L-arginine dehydrogenase (EC 1.4.1.25, L-ArgDH) is one of the most

5 recently discovered and is known to physiologically function in concert with FAD-dependent D-

6 arginine dehydrogenase for the conversion (epimerization) of D-arginine to L-arginine via its oxo-

7 analog, 5-guanidino-2-oxopentanoate, in Psuedomonas aeruginosa POA1 [6]. The gene DauB in $P$.

8 aeruginosa has been expressed in Escherichia coli, and the product reportedly catalyzes the

9 reversible deamination of L-arginine to 5-guanidino-2-oxopentanoate in the presence of NAD(P).

10 However, the molecular and catalytic properties of L-ArgDH have not yet been reported.

We previously identified a putative L-ArgDH gene, PverR02_12350, within the genome of the nonpathogenic bacterium P. veronii (KEGG Organisms: Complete Genomes: www.genome.jp/kegg/catalog/org_list. Html). This gene possesses high amino acid sequence

14 homology (67.8\%) with a gene encoding L-ArgDH in the pathogenic bacterium P. aeruginosa

15 POA1. Here, we succeeded in the expressing the P. veronii JCM 11942 gene in E. coli, using artificial primers synthesized from the $\mathrm{N}$ - and $\mathrm{C}$-terminal sequences inferred from the putative

17 L-ArgDH gene. The expressed product was purified, characterized, and applied to a simple colorimetric assay for L-arginine. 
$2 \quad$ Materials and methods

\section{$3 \quad$ Materials}

4 L-Arginine, NADP and NAD were purchased from Wako Pure Chemical Corporation (Tokyo,

5 Japan). Methoxy-phenasine methosulfate (m-PMS), water soluble tetrazolium 1 (WST-1) and 2-(4-

6 iodophenyl)-3-(4-nitrophenyl)-5-phenyl-2H-tetrazolium chloride (INT) were from Chemical Dojin

7 Co., Ltd. (Kumamoto, Japan). Yeast extract and Luria-Bertani (LB) medium were from Becton,

8 Dickinson and Company (Sparks, MD, USA), and hipolypepton S was from Nihon Pharmaceutical

9 Co., Ltd. (Tokyo). P. veronii JCM 11942 was obtained from the Japan Collection of Microorganisms

10 (Wako, Saitama, Japan). Ni Sepharose 6 First Flow resin was purchased from Cytiva (Tokyo, Japan).

11 Infusion vector was obtained from Takara Bio Inc. (Kusatsu, Shiga, Japan). All other chemicals were

12 of reagent grade.

14 Cloning a putative ArgDH gene from P. veronii JCM 11942 and determination of the gene

15 sequence

16 P. veronii JCM 11942 was aerobically grown in the medium $(150 \mathrm{~mL}$ in a $0.5 \mathrm{~L}$ flask $)$ containing

$170.5 \%$ hipolypepton, $0.3 \%$ beef extract, and $0.5 \% \mathrm{NaCl}$ for $24 \mathrm{~h}$ at $30^{\circ} \mathrm{C}$, after which the cells $1.3 \mathrm{~g}$

18 (wet weight) were harvested by centrifugation. The cells were homogenized using a Multi-beads 
1 Shocker (Yasui Kikai, Osaka, Japan), and the crude DNA extract was prepared using the phenol-

2 chloroform-isoamyl alcohol method. To remove RNA, the DNA extract was treated with RNase A,

3 after which the genomic DNA was purified using phenol-chloroform-isoamyl alcohol. The L-ArgDH

4 gene was amplified by PCR using the genomic DNA as the template with the primers 5'-

5 GGAGATATACATATGTCCAGCACGCCCCATGTCATC-3' (forward) and 5'-

6 GCTCGAATTCGGATCGCGCTGCAGCTGGTACAGCG-3' (reverse). The forward and reverse

7 primers contained the sequence of pET21a (underlined) and the putative ArgDH gene, PverR02_12350,

8 queried from the Genome Net database. The expression vector pET21a was linearized using inverse

9 PCR with the primers 5'-CATATGTATATCTCCTTCTTAAAGTTAAACAAAATTATTT-

10 CTAGAGGGG-3' (forward) and 5'-GATCCGAATTCGAGCTCCGTCGACAAG-3' (reverse). The

11 amplified target gene fragments were ligated into the linearized pET21 a vector using an In-Fusion HD

12 cloning kit (Takara Bio Inc.). The sequence of the L-ArgDH gene inserted into pET21a was

13 determined by Hokkaido System Science (Hokkaido, Japan).

15 Growth conditions for $E$. coli BL21(DE3) transformed with pET21a-L-ArgDH and overexpression of L-ArgDH

17 E. coli BL21(DE3) was transformed with the prepared expression vector (pET21a-L-ArgDH) and

18 cultured to logarithmic phase $\left(\mathrm{OD}_{660}=0.5\right)$ at $37^{\circ} \mathrm{C}$ in $150 \mathrm{~mL}$ of $\mathrm{LB}$ medium containing 0.1 
1

$\mathrm{mg} / \mathrm{mL}$ ampicillin. IPTG (final $0.1 \mathrm{mM}$ ) was then added to the culture medium, and the incubation

was continued for an additional $3 \mathrm{~h}$ at $37^{\circ} \mathrm{C}$. The cells were then collected by centrifugation and stored at $-80^{\circ} \mathrm{C}$ until use.

\section{Activity and protein assays}

The standard assay for L-ArgDH activity was performed at $30^{\circ} \mathrm{C}$ and entailed measuring the initial rate of NADPH formation from NADP and L-arginine. The reaction mixture consisted of $0.2 \mathrm{M}$ glycine-KOH buffer (pH 9.5), 10 mM L-arginine ( $\mathrm{pH} 9.5$ ), $1.25 \mathrm{mM}$ NADP and the enzyme in a final volume of $1.00 \mathrm{~mL}$. The mixture solution $(0.96-0.99 \mathrm{~mL})$ without enzyme was incubated at $30^{\circ} \mathrm{C}$ for about $3 \mathrm{~min}$ in a cuvette with 10 -mm light path. The reaction was then started by addition of enzyme (0.01-0.04 mL). The initial increase in absorbance at $340 \mathrm{~nm}$ was measured under temperaturecontrolled cell conditions at $30^{\circ} \mathrm{C}$. One unit of enzyme was defined as the amount of enzyme producing $1 \mu \mathrm{mol}$ of NADPH per minute at $30^{\circ} \mathrm{C}$. The absorption coefficient $\left(6.2 \mathrm{mM}^{-1} \mathrm{~cm}^{-1}\right)$ of NADPH at 340 $\mathrm{nm}$ was used. Protein concentrations were calculated from the absorbance at $280 \mathrm{~nm}(1.0 \mathrm{mg} / \mathrm{mL}=0.80$ obtained from the amino acid composition of $\mathrm{L}-\mathrm{ArgDH})$. Replicability was ensured by making the measurements triplicate.

\section{Enzyme purification}


1 E. coli cells expressing the recombinant enzyme (wet weight: about $1.3 \mathrm{~g}$ wet weight obtained from a

$2150 \mathrm{~mL}$ culture medium) were suspended in $20 \mathrm{mM} \mathrm{NaH}_{2} \mathrm{PO}_{4}-\mathrm{Na}_{2} \mathrm{PO}_{4}$ buffer (pH 7.9) solution $(6 \mathrm{~mL})$

3 containing $10 \%$ glycerol, $0.5 \mathrm{M} \mathrm{NaCl}, 0.01 \%$ 2-mercaptethanol and $5 \mathrm{mM}$ imidazole, and disrupted

4 by sonication. The crude extract (about $7 \mathrm{~mL}$ ) was prepared as the supernatant clarified by 5 centrifugation $(12,000 \mathrm{~g} \times 10 \mathrm{~min})$ and applied on a Ni Sepharose High Performance column $(\varphi 10 \mathrm{x}$

$6 \quad 66 \mathrm{~mm}$ ). The column was washed first with the same buffer solution (about $36 \mathrm{~mL}$ with a 3-mL

7 fraction) and then with the same buffer solution $(30 \mathrm{~mL})$ but with $60 \mathrm{mM}$ imidazole. The active

8 enzyme was eluted with $20 \mathrm{mM} \mathrm{KH} \mathrm{PO}_{4}-\mathrm{K}_{2} \mathrm{HPO}_{4}$ buffer solution ( $\mathrm{pH} 7.2$, about $40 \mathrm{~mL}$ ) containing

$910 \%$ glycerol, $0.5 \mathrm{M} \mathrm{NaCl}, 0.01 \%$ 2-mercaptethanol and $400 \mathrm{mM}$ imidazole. The active enzyme

10 solution was dialyzed against $20 \mathrm{mM} \mathrm{KH}_{2} \mathrm{PO}_{4}-\mathrm{K}_{2} \mathrm{HPO}_{4}$ buffer (pH 7.2) containing $10 \%$ glycerol and

$11 \quad 0.01 \%$ 2-mercaptethanol and then stored in refrigerator $\left(4^{\circ} \mathrm{C}\right.$ or $\left.-20^{\circ} \mathrm{C}\right)$ before use.

\section{Effects of glycerol and pH on enzyme stability}

14 The recombinant $E$. coli cells were suspended in $20 \mathrm{mM} \mathrm{Na} 2 \mathrm{HPO}_{4}-\mathrm{NaH}_{2} \mathrm{PO}_{4}$ buffer (pH 7.2)

supplemented with $0.01 \%$ 2-mercaptoethnol and disrupted by sonication with or without addition of

$10 \%$ glycerol. After centrifugation $(12,000 \mathrm{~g}$ for $10 \mathrm{~min})$, the supernatant was stored at $4{ }^{\circ} \mathrm{C}$ and the

time-dependent changes in L-ArgDH activity were monitored. 
$1 \mathrm{pH}$ buffer solutions (50 mM, pH 6-9.5) containing $10 \%$ glycerol and 0.01\% 2-MET, after which the

2 activity of an aliquot $(10 \mu \mathrm{L})$ was measured under the standard assay conditions for NADP-dependent

3 L-ArgDH formation.

4

$5 \quad$ Effect of $\mathbf{p H}$ and temperature on enzyme activity

6 Enzyme activity was measured in different $\mathrm{pH}$ buffers under the standard assay conditions. The buffers

7 used were $0.2 \mathrm{M} \mathrm{K}_{2} \mathrm{HPO}_{4}-\mathrm{KH}_{2} \mathrm{PO}_{4}(\mathrm{pH}$ 6.8-8.5) and glycine-KOH (pH 9-10.5). To evaluate the effect

8 of temperature on its stability, the enzyme (in $20 \mathrm{mM} \mathrm{K}_{2} \mathrm{HPO}_{4}-\mathrm{KH}_{2} \mathrm{PO}_{4}$ buffer, $\mathrm{pH} 7.5$, containing

$9 \quad 10 \%$ glycerol and $0.01 \%$ 2-MET) was incubated for $30 \mathrm{~min}$ at different temperatures, after which the

remaining activity was measured under the standard assay conditions.

Polyacrylamide electrophoresis (PAGE)

13 Native-PAGE (disc gel) was carried out at $4^{\circ} \mathrm{C}$ on a $7.5 \%$ polyacrylamide gel, as previously

14 described [7]. The protein was then stained using $0.025 \%$ Coomassie brilliant blue R-250 in 50\%

15 methanol and $10 \%$ acetate. In addition, active staining was performed at $30^{\circ} \mathrm{C}$ using a reaction

mixture (5.0 mL) containing 0.20 M K2 $\mathrm{HPO}_{4}-\mathrm{KH}_{2} \mathrm{PO}_{4}$ buffer (pH 8.0), $1.0 \mathrm{mM}$ NADP, $0.2 \mathrm{mM} \mathrm{m}$ -

PMS, $1 \mathrm{mM}$ INT, and $10 \mathrm{mM} \mathrm{L-arginine} \mathrm{(pH} \mathrm{8.0)} \mathrm{or} \mathrm{other} \mathrm{amino} \mathrm{acids} \mathrm{(} \mathrm{pH}$ 8.0) until a red band of 
1 PAGE was carried out on $12.5 \%$ polyacrylamide gel using the method of Laemmli [8]. Precision

2 Plus protein standards (Bio-Rad Laboratories, CA, USA) were used as the molecular mass standards.

3 The protein sample was boiled for $5 \mathrm{~min}$ in $10 \mathrm{mM}$ Tris- $\mathrm{HCl}$ buffer (pH 7.0) containing 1\% SDS and

$4 \quad 1 \% 2$-mercaptoethanol. Protein bands were visualized by staining with $0.025 \%$ Coomassie brilliant

5 blue R-250 in 50\% methanol and $10 \%$ acetate.

6

\section{$7 \quad$ Substrate specificity and kinetic parameters}

8 The ability of amino acids to serve as electron donors was assessed based on activity staining on native

9 disc PAGE. Activity staining was performed as described above with various amino acids $(10 \mathrm{mM}$,

$10 \mathrm{pH}$ 8) other than L-arginine in the assay mixture. To determine the Michaelis constants $\left(K_{\mathrm{m}}\right)$ for L-

11 arginine and $\mathrm{NAD}(\mathrm{P})$, initial velocity analyses were carried out under the standard assay conditions,

after which the $K_{\mathrm{m}}$ values for the substrates were determined using double reciprocal plots of initial

\section{Molecular mass determination}

The molecular mass of the native enzyme was determined by gel filtration column chromatography

using a Superdex $200 \mathrm{pg}$ column $(2.6 \times 60 \mathrm{~cm})$. Ferritin $(440 \mathrm{kDa})$, aldolase $(158 \mathrm{kDa})$, conalbumin

(75 kDa), ovalbumin (43 kDa) and $\alpha$-chymotrypsinogen ( $25 \mathrm{kDa})$ served as molecular standards (GE 
1 Healthcare). Subunit molecular mass was determined by SDS-PAGE (12.5\% acrylamide slab gel, 1

$2 \quad$ mm thick) using the same marker proteins (Takara Bio).

3

\section{Sequence alignments}

5 The amino acid sequences of L-ArgDHs and the putative enzyme were collected from the KEGG

6 database (https://www.genome.jp/kegg/) and aligned using Clustal W multiple sequence alignment

7 software (ver. 2.1) [9] via the DNA Data Bank of Japan (DDBJ) website

8 (https://www.ddbj.nig.ac.jp/index.html).

\section{Determination of L-arginine using L-ArgDH}

11 The enzymatic method for determining L-arginine levels using L-ArgDH consists of two reaction steps.

The first step is the quantitative conversion of L-arginine to its oxo analog and ammonia with conversion of NADP to NADPH by L-ArgDH. The reaction mixture $(0.40 \mathrm{~mL})$ consisted of $100 \mathrm{mM}$ glycine-KOH buffer (pH 9.5), $2.5 \mathrm{mM}$ NADP, the enzyme (0.42 U) and L-arginine ( $\leqq 100 \mu \mathrm{M})$. The second step is the chemical conversion of NADPH to reduced WST-1 (formazan) via a redox mediator (m-PMS). The reaction mixture was incubated at $30^{\circ} \mathrm{C}$ for $10 \mathrm{~min}$ and then $400 \mathrm{mM} \mathrm{K} 2 \mathrm{HPO}_{4}-\mathrm{KH}_{2} \mathrm{PO}_{4}$ 
4

were added giving a final volume of $1.00 \mathrm{~mL}$. The formazan formed from WST-1 was determined by measurement of absorbance at $438 \mathrm{~nm}$.

西

\section{Results and Discussion}

\section{Expression, stabilization, and purification of NADP-dependent L-ArgDH}

A putative gene encoding L-ArgDH in the nonpathogenic bacterium P. veronii JCM 11942 was

successively expressed in $E$. coli cells, after which the cell extract exhibited strong NADP-dependent

L-arginine dehydrogenase activity. However, the enzyme in the crude extract containing $20 \mathrm{mM}$

phosphate buffer, $\mathrm{pH} 7.9$ or 7.2, supplemented with $0.01 \%$ 2-MET was somewhat unstable, and nearly

all activity was lost within 2 days (Fig. 1). We therefore evaluated the stabilizing effect of adding

glycerol to the enzyme solution and found that the enzyme was greatly stabilized by the addition of

$10 \%$ glycerol to the crude extract (Fig. 1). In the presence of $10 \%$ glycerol, no loss of enzyme activity

was observed for at least one month at temperatures of $4^{\circ} \mathrm{C}$ or $-20^{\circ} \mathrm{C}$. The enzyme was therefore stored

in $20 \mathrm{mM}$ phosphate buffer (pH 7.2) supplemented with $10 \%$ glycerol and $0.01 \%$ 2-MET unless

otherwise stated. The enzyme was then effectively purified to homogeneity (Fig. 2) with a high yield

using His-tagged affinity chromatography (Table 1). 


\section{$1 \quad$ Enzymological properties}

2 Using gel filtration chromatography, the molecular mass of the native enzyme was determined to be

$366 \mathrm{kDa}$. In addition, SDS-PAGE gave a single band, and the subunit molecular mass of was calculated

4 to be $34 \mathrm{kDa}$. This indicates the enzyme consists of dimeric structure with two identical subunits.

5 The ability for L-arginine and several other amino acids to serve as electron doners was evaluated

6 based on activity staining on native-PAGE. When L-arginine was used as the electron doner, a distinct

7 activity band was detected, and the mobility was identical to that of the protein staining band (Fig.

8 2). By contrast, no activity band was observed after activity staining on native-PAGE with D-arginine,

9 L-lysine, L-ornithine, L-citrulline, L-leucine, L-phenylalanine, L-histidine, L-glutamate, glycine or

10 L-alanine. This means that this enzyme is highly selective for L-arginine. As the electron acceptor,

11 NADP was much more preferable than NAD; the reaction rate for NADP was about 4 times higher than that for NAD under the standard assay conditions. This is in contrast to the findings of Lie and $\mathrm{Lu}$ [6], who reported that using P. aeruginosa L-ArgDH, the reaction rate with NADP was nearly the same as with NAD. incubation for $30 \mathrm{~min}$, the enzyme retained all of its activity at temperatures up to $35^{\circ} \mathrm{C}$, but it lost

17 more than $90 \%$ of its activity at $45^{\circ} \mathrm{C}$ (Fig. 3). In addition, the enzyme activity increased with 
1 in the presence of $10 \%$ glycerol and $0.01 \%$ 2-MET (Fig. 4), but the activity markedly declined below

2 pH 6.5 or above pH 9 (Fig. 4). From the analysis of the pH effect on L-arginine oxidation, the

3 maximum activity was observed at around $\mathrm{pH} 9.5$ (Fig. 5A). Similarly high optimum pHs for activity

4 were previously observed with other amino acid dehydrogenases, including alanine, glutamate,

5 phenylalanine, leucine and tryptophan dehydrogenases $[1,11]$

Plots of the initial velocity versus both L-arginine and NADP concentrations gave typical

Michaelis-Menten type kinetics. Using double reciprocal plots of the initial velocities and substrate

concentrations, the apparent $\mathrm{Km}$ values for L-arginine and NADP were determined to be $2.5 \mathrm{mM}$ and

$0.20 \mathrm{mM}$, respectively.

After determining the gene sequence of $P$. veronii JCM 11942 L-ArgDH, the deduced amino

11 acid sequence (315 amino acid residues) was aligned with those of $P$. aeruginosa PO1 and the

putative P. veronii L-ArgDHs (Fig. 6). Of the 315 amino acid residues comprising P. veronii JCM

11942 L-ArgDH, only two, A42 and N95, differed from those (P42 and S95, respectively) of the

putative P. veronii PverR02_12350 enzyme (sequence homology: 99.4\%). By contrast, the sequence

of $P$. veronii JCM 11942 L-ArgDH had only $67.8 \%$ homology with the P. aeruginosa PAO1 enzyme,

which is rather low for an enzyme from the same Pseudomonas family. In addition, a phylogenetic

17 tree prepared using the Clustal $\mathrm{W}$ data base with the amino acid sequences of L-ArgDHs in the

18 Psuedomonas family showed that $P$. veronii $\mathrm{L}-\mathrm{ArgDH}$ formed a different cluster from that of the $P$. 
1

2

3

4

6

aeruginosa enzyme (Fig. 7). This is consistent with P. veronii L-ArgDH exhibiting different catalytic properties than the $P$. aeruginosa enzyme. As mentioned, for example, $P$. veronii $\mathrm{L}-\mathrm{ArgDH}$ is much more selective for NADP over NAD as the electron acceptor, whereas the P. aruginosa PAO1 enzyme utilizes NADP and NAD equally [6]. This tree shows that ArgDH widely distributes among Pseudomonas species, and we are now investigating the three-dimensional structure of $P$. veronii JCM 11942 L-ArgDH as a new amino acid dehydrogenase.

(1)

\section{L-Arginine determination using L-Arg DH}

The simple colorimetric assay for L-arginine consisted of two steps because of the different optimum $\mathrm{pHs}$ for enzymatic NADPH formation with L-arginine catalyzed by L-ArgDH $(\mathrm{pH} 9.5)$ and the reduction of WST1 to formazan with NADPH ( $\mathrm{pH} \leqq 8.0)$. A linear relationship was obtained between the L-arginine concentration $(\leqq 100 \mu \mathrm{M})$ and absorbance at $438 \mathrm{~nm}$ (Fig. 8). This colorimetric method is simple, rapid (the reaction time $\leqq 10 \mathrm{~min}$ ) and highly specific for L-arginine. The method is applicable to many food and clinical analyses because L-arginine is an intermediate in the human urea-cycle and is an essential amino acid in humans. Although a colorimetric method for assaying L-arginine using five different enzymes has been reported [12], our simple method for assaying L-arginine may be much more attractive for industrial and clinical uses. 
1

2 We found the gene homolog of L-ArgDH in genome data of Pseudomonas veronii. The P. veronii

3 JCM11942 gene was successively expressed in E. coli, and the gene product exhibited strong NADP-

4 dependent L-ArgDH activity. The enzyme was unstable but was stabilized by the presence of $10 \%$

5 glycerol under neutral $\mathrm{pH}$ conditions. The enzyme was purified to homogeneity through a single Ni-

6 chelate affinity chromatography step and consisted of a homodimeric protein with a molecular mass

7 of about $65 \mathrm{kDa}$. The enzyme selectively catalyzed L-arginine oxidation in the presence of NADP,

8 with maximal activity at $\mathrm{pH}$ 9.5. The apparent $K_{\mathrm{m}}$ values for L-arginine and NADP were 2.5 and

$90.21 \mathrm{mM}$, respectively. Furthermore, A simple colorimetric microassay for L-arginine was achieved

10 using the enzyme.

11

12

\section{References}

13

1. Ohshima, T. \& Soda, K. (2000). in Stereoselective biocatalysis: amino acid dehydrogenases and their applications. "Stereoselective Biocatalysis" (Patel R. N., ed.), Marcel Dekker, Inc. (New York/Basel), pp. 877-902. ISBN : 9780367801250.

2. Akita, H., Hayasi. J., Sakuraba, H., \& Ohshima, T. (2018). Artificial thermostable D-amino acid dehydrogenase: creation and application. Front. Microbiol. 2018, 2018 Aug 3;9:1760. doi: 10.3389/fmicb.2018.01760. 
1 3. Dave, U. C., \& Kadeppagari, R. K. (2019). Alanine dehydrogenase and its applications- A review.

Clin. Rev. Biotechnol. 39, 648-654. doi: 10.1080/07388551.2019.1594153.

4. Luo, W., Zhu, J., Zhao, Y., Zhang, H., Yang, X., Liu, Y., Rao, Z., \& Yu, X. (2020). Cloning and expression of a novel leucine dehydrogenase: Characterization and L-tert-leucine production. Front Bioeng. Biotechnol. 2020 Mar 31, 8, 186. doi: 10.3389/fbioe.2020.00186.

5. Wendel, U,, Hummel, W., \& Langenbech, U. (1989). Monitoring of phenylketonuria: A colorimetric method for the determination of plasma phenylalanine using L-phenylalanine dehydrogenase. Anal. Biochem. 180:91-94. doi: 10.1016/0003-2697(89)90092-4

6. Li, C., \& Lu, C. -D. (2009). Arginine racemization of by coupled catabolic and anabolic dehydrogenases. Proc. Natl. Acad. Sci. 906-911. doi:10.1073/pnas.0808269106.

7. Akita, H., Fujino, Y., Doi, K., \& Ohshima, T, (2011). Highly stable meso-diaminopimelate dehydrogenase from an Ureibacillus thermosphaericus strain A1 isolated from a Japanese compost: purification, characterization and sequencing. AMS Express 1(1)43._doi: 10.1186/21910855-1-43.

8. Laemmli, U. K. (1970). Cleavage of structural proteins during the assembly of the head of bacteriophage T4. Nature. 1970, 227:680-685. doi: 10.1038/227680a0.

9. Thompson, D. J., Higgins, D. G., \& Gibsonet, T.J., (1994). CLUSTAL W: improving the sensitivity of progressive multiple sequence alignment through sequence weighting, position- 
1

2

specific gap penalties and weight matrix choice, Nucleic Acids Res. 22, 4673-4680. doi:

$10.1093 / \mathrm{nar} / 22.22 .4673$

10. Perriére, G., \& Gouy, M., (1996). WWW-query: an on-line retrieval system for biological sequence banks. Biochimie. 78, 364-369. doi: 10.1016/0300-9084(96)84768-7.

11. Ogura, R., Wakayama, T., Mutaguchi, Y., Doi, K., \& Ohshima, T. (2014). Biochemical characterization of an L-tryptophan dehydrogenase from the photoautotrophic cyanobacterium Nostoc punctiforme. Enzyme Microb. Technol. 60, 40-46. doi: 10.1016/j.enzmictec.2014.04.002

12. Kameya, M., \& Asano, Y. (2014) Rapid enzymatic assays for L-citrulline and L-arginine based on the platform of pyrophosphate detection. Enzyme Microb. Technol. 57, 36-41. doi: 10.1016/ j.enzmictec.2014.01.008.

\section{Statements and declarations}

Funding This work was supported in part by research funding from the Japan Society for the Promotion of Science KAKENHI grant No. 20K05816 to T. O.

\section{Competing interest}

The authors declare that they have no competing interests.

Author contribution All authors contributed to the study conception and design, and performed 
1 material preparation, data collection and analysis. Toshihisa Ohshima mainly wrote the manuscript in

2 discussion during the preparation of the manuscript.

3

4

5

6

7

8

9

10

11

12

13

14

15

16

17

18 
1 Table 1. Summary of L-ArgDH purification

2

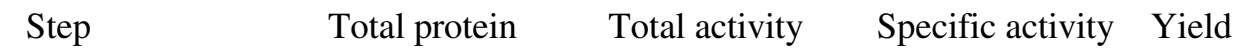

4

$\begin{array}{llll}(\mathrm{mg}) & \text { (units) } \quad \text { (units/mg) }\end{array}$

5

Crude extract

95.2

153

1.61

100

$6 \quad$ Ni-chelate affinity

7 chromatography

19.9

61.4

3.09

40

8

9

10

11

12

13

14

15

16

17

18

19

20 
2 Fig. 1. Effect of glycerol addition on L-ArgDH storage. L-ArgDH was stored at $4^{\circ} \mathrm{C}$ in $20 \mathrm{mM}$

$3 \quad \mathrm{H}_{2} \mathrm{PO}_{4}-\mathrm{K}_{2} \mathrm{HPO}_{4}$ phosphate buffer $(\mathrm{pH}$ 7.2) containing 0.01\% 2-MET with (•) or without $(\boldsymbol{\bullet}) 10 \%$

4 glycerol. Aliquots $(10 \mu \mathrm{L})$ of the enzyme solution are depicted, and the activity was measured under

$5 \quad$ the standard assay conditions $(\mathrm{n}=3)$.

6

7 Fig. 2. Native disc-PAGE of purified L-ArgDH. The enzyme (about $125 \mu \mathrm{g} /$ tube) was applied on a 8 disc gel and the gel was stained with CBB (A) or from L-arginine activity (B) after electrophoresis.

9

\section{Legends to Figures}

Fig. 3. Effect of temperature on L-ArgDH stability. Solution containing the purified enzyme (20 mM $\mathrm{KH}_{2} \mathrm{PO}_{4}-\mathrm{K}_{2} \mathrm{HPO}_{4}$ buffer, $\mathrm{pH} 7.2$, containing $10 \%$ glycerol and $0.01 \%$ 2-ME) was incubated for 30 min at different temperatures. The activity remaining in aliquots $(10 \mu \mathrm{L})$ of the solution was then measured under the standard assay conditions. $(n=3)$

Fig. 4. Effect of $\mathrm{pH}$ on L-ArgDH stability. The enzyme was incubated in $50 \mathrm{mM}$ buffer solutions

$\left(10 \%\right.$ glycerol $+0.01 \%$ 2-ME) with different $\mathrm{pHs}$ for $30 \mathrm{~min}$ at $30^{\circ} \mathrm{C}$. The activity remaining in aliquots $(10 \mu \mathrm{L})$ of the solution was the measured under the standard assay conditions. The buffers used were $\mathrm{KH}_{2} \mathrm{PO}_{4}-\mathrm{K}_{2} \mathrm{HPO}_{4}(\mathrm{pH} 6.0-8.5)$ and glycine- $\mathrm{KOH}$ (pH 9.0-10). (n=3) 
2 Fig. 5. Effects of temperature (A) and pH (B) on L-ArgDH activity. Enzyme activity was

3 measured at different temperatures (A) and in different $\mathrm{pH}$ buffers (B) under the standard assay

4 conditions. The buffers used in (B) were $0.2 \mathrm{M} \mathrm{KH}_{2} \mathrm{PO}_{4}-\mathrm{K}_{2} \mathrm{HPO}_{4}(\mathbf{\bullet})$, Tris- $\mathrm{HCl}(\boldsymbol{\Delta})$, and Gly-NaOH

$7 \quad$ Fig. 6. Amino acid sequence of $P$. veronii JCM 11942 L-ArgDHand the alignments with the

8 sequences of the putative P. veronii Pver_12550 and P. aeruginosa PA01_PA3862 L-ArgDHs. (•):

9 Amino acid residues in the sequence of $P$. veronii JCM 11942 L-ArgDH that differed from those in

10 the sequence of the putative P. veronii Pver_12550 L-ArgDH. ( $\rightarrow$ ): Adenine binding motif

11 (GXGXXA(G)) for NADP. The sequence added as a his-tag at the C-terminus of P. veronii

JCM 11942-L-ArgDH is DPNSSSVDKLAAALEHHHHHH. The sequence alignment was prepared using Clustal W [9] .

L-ArgDH and homologous proteins from Pseudomonas and Burkholderia strains. The phylogenetic 
$1 \quad$ P.: Pseudomonas and B.: Burkholderia. Rectangles show P. veronii JCM 11942 and P. aeromonas

2 PAO1 L-ArgDHs; the others are putative ornithine cyclodeaminase or L-ArgDH.

3

4 Fig. 8. Calibration curve for L-arginine determination in an assay using L-ArgDH. Oxid and Red

5 m-PMS: methoxy-phenagine methosulfate oxidized and reduced forms, respectively. WST-1 water

6 soluble tetrazolium.

7

8

9

10 


\section{Figures}

\section{Figure 1}

Effect of glycerol addition on L-ArgDH storage. L-ArgDH was stored at $4 \mathbb{\text { in }} 20 \mathrm{mM} \mathrm{H2PO4-K2HPO4}$

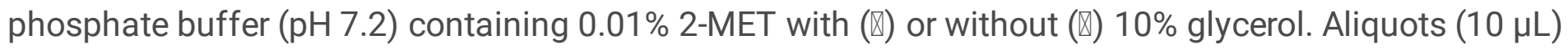
of the enzyme solution are depicted, and the activity was measured under the standard assay conditions $(n=3)$.

\section{Figure 2}

Native disc-PAGE of purified L-ArgDH. The enzyme (about $125 \mu \mathrm{g} / \mathrm{tube}$ ) was applied on a disc gel and the gel was stained with CBB (A) or from L-arginine activity (B) after electrophoresis.

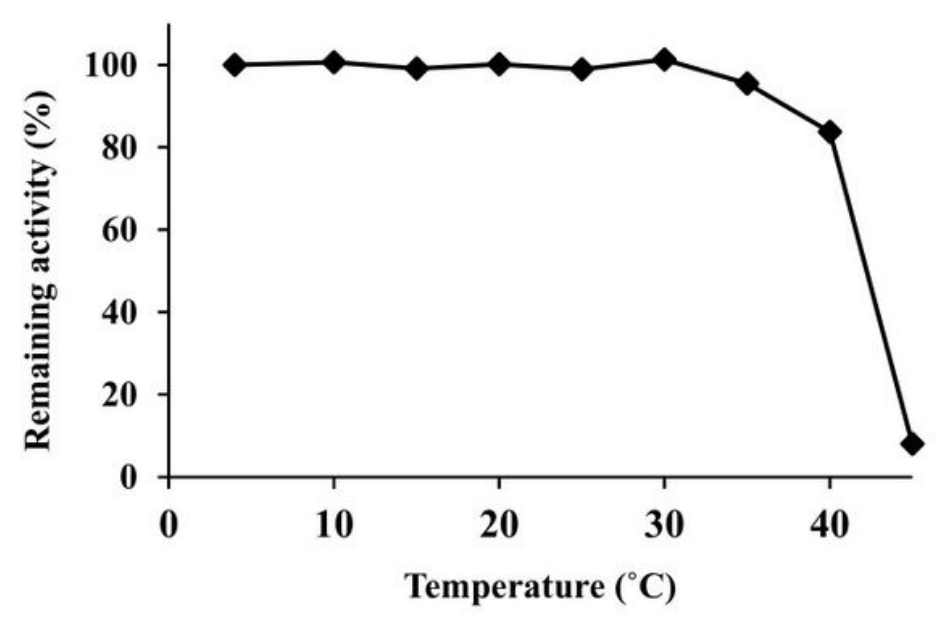

Fig. 3 .

T. Ohshima et al.

\section{Figure 3}

Effect of temperature on L-ArgDH stability. Solution containing the purified enzyme (20 mM KH2PO4K2HPO4 buffer, $\mathrm{pH} 7.2$, containing $10 \%$ glycerol and 0.01\% 2-ME) was incubated for $30 \mathrm{~min}$ at different 
temperatures. The activity remaining in aliquots $(10 \mu \mathrm{L})$ of the solution was then measured under the standard assay conditions. $(n=3)$

\section{Figure 4}

Effect of pH on L-ArgDH stability. The enzyme was incubated in $50 \mathrm{mM}$ buffer solutions (10\% glycerol $+0.01 \%$ 2-ME) with different pHs for $30 \mathrm{~min}$ at 30区. The activity remaining in aliquots (10 $\mu \mathrm{L})$ of the solution was the measured under the standard assay conditions. The buffers used were $\mathrm{KH} 2 \mathrm{PO} 4-$ K2HPO4 (pH 6.0-8.5) and glycine-KOH ( $\mathrm{pH} 9.0-10) .(n=3)$

\section{Figure 5}

Effects of temperature (A) and pH (B) on L-ArgDH activity. Enzyme activity was measured at different temperatures $(A)$ and in different $\mathrm{pH}$ buffers $(B)$ under the standard assay conditions. The buffers used in (B) were 0.2 M KH2PO4-K2HPO4 (『), Tris-HCl (『), and Gly-NaOH (区).

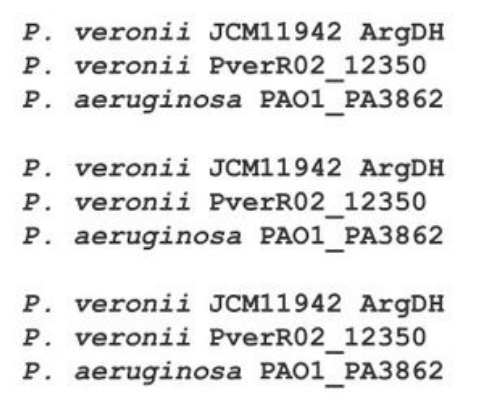

P. veronii JCM11942 ArgDH

P. veronii PverR02_12350

P. aeruginosa PAO1_PA3862

P. veronii JCM11942 ArgDH

P. veronii PverR02_ 12350

P. aeruginosa PAO1_PA3862

P. veronii JCM11942 ArgDH

P. vronii PverR02_12350J

P. aeruginosa PAOI_PA3862

P. veronii JCM11942 ArgDH

Fig. 6

P. veronii PverR02_12350

P. aeruginosa PAO1_PA3862
1 -MSSTPHVIQQAQARELLAQIDVPQILHKLFRDLAAGLAVQPÅQQLVAFP 49

1 -MSSTPHVIQQAQARELLAQIDVPQILHKLFRDLAAGLAVQPPQQLVAFP 49

1 MSAATPLIVQQAEAEQLLARIDVLQAMRQLFLDLAAGQALQPAQQLVEFP 50 KGAGDFINYLGVLAEDGVYGVKTSPYIVGEQGPLVTAWTLLMSMHNGQPL 99 KGAGDFINYLGVLAEDGVYGVKTSPYIVGEQGPLVTAWTLLMSMHSGQPL 99 AGRGDFINYLGVLAQEQVYGVKTSPYIVREQGPLVTAWTLLMSMQTGQPL 100

LLCDAHELTTARTAATTALAVDALAPLAARRLAI I GSGKVAQAHLRYVQN 149 LLCDAHELTTARTAATTALAVDALAPLAARRLAIIGSGKVAQAHLRYVQN 149 LLCDAARLTTARTAATTAVAVDALAPAEACRLALIGSGPVAHAHLQYVKG 150

LRDWQHISLFSPSLASASPATLAQLTGLDPRLSIADSCAAAVADADVIML 199 LRDWQHISLFSPSLASASPATLAQLTGLDPRLSIADSCAAAVADADVIML 199 LRDWQGVRVHSPCLDERR---LQSLRAIDPRAEAAGSLEEALDEADVILL 197

CTSSAGPVLDPAHLSKPALITSISTNAPRAHEVPPHSLNAMQVFCDYRQT 249 CTSSAGPVLDPAHLSKPALITSISTNAPRAHEVPPHSLNAMQVFCDYRQT 249 CTSSARAVIDPRQLKRPALVTSISTNAPRAHEVPAESLAAMDVYCDYRHT 247

TPDAAGEMLIASEQHGWDKRAVMGDLPELLSDMAQRPDYQRPVFFRSIGL 299 TPDAAGEMLIASEQHGWDKRAVMGDLPELLSDMAQRPDYQRPVFFRSIGL 299 TPGSAGEMLIAAEQHGWSPEAIRGDLAELLSAQAPRPEYRRPAFFRSIGL 297

GLEDIALANALYQLQR- $315100 \%$

GLEDIALANALYQLQR- $315 \quad 99.4 \%$

GLEDVALANALYRLRQAG $315 \quad 67.0 \%$

T. Ohshima et al.

\section{Figure 6}

Amino acid sequence of P. veronii JCM $11942 \mathrm{~L}-\mathrm{ArgDH}$ and the alignments with the sequences of the putative P. veronii Pver_12550 and P. aeruginosa PA01_PA3862 L-ArgDHs. (区): Amino acid residues in the 
sequence of P. veronii JCM $11942 \mathrm{~L}$-ArgDH that differed from those in the sequence of the putative $\mathrm{P}$. veronii Pver_12550 L-ArgDH. (-): Adenine binding motif (GXGXXA(G)) for NADP. The sequence added as a his-tag at the C-terminus of P. veronii JCM 11942-L-ArgDH is DPNSSSVDKLAAALEHHHHHH. The sequence alignment was prepared using Clustal W [9] .

\section{Figure 7}

Condensed phylogenetic tree based on the amino acid sequences of P. veronii JCM 12942 L-ArgDH and homologous proteins from Pseudomonas and Burkholderia strains. The phylogenetic tree was prepared using Clustal W [9] 1994) and NJ plot (Perriére and Gouy, 1996). Abbreviations: P.: Pseudomonas and B.: Burkholderia. Rectangles show P. veronii JCM 11942 and P. aeromonas PAO1 L-ArgDHs; the others are putative ornithine cyclodeaminase or L-ArgDH.

\section{Figure 8}

Calibration curve for L-arginine determination in an assay using L-ArgDH. Oxid and Red m-PMS: methoxyphenagine methosulfate oxidized and reduced forms, respectively. WST-1 water soluble tetrazolium. 